\title{
Definiciones e imágenes del concepto de ángulo y su medida en estudiantes que inician la educación superior
}

\author{
Definitions and images of the concept of angle and its \\ measurement among students who are beginning their \\ undergraduate studies
}

\section{Yanira Pachuca Herrera ${ }^{1}$ \\ Gonzalo Zubieta Badillo}

\begin{abstract}
Resumen: El título de este escrito provoca a priori desconcierto, pues el lector podría considerar que el concepto de ángulo y su medida, está superado por los estudiantes al iniciar una licenciatura, no obstante, aunque algunas dificultades reportadas en la literatura si lo están, otras todavía persisten. En matemáticas la definición de un concepto resulta relevante, sin embargo, en la práctica los estudiantes recurren usualmente a la imagen evocada del concepto, lo que con frecuencia les trae dificultades para realizar una tarea específica. Se diseñó un cuestionario basado en la noción de ángulo y su medida. Los ítems fueron contestados por 22 estudiantes mexicanos del primer semestre de la Licenciatura en Física y Matemáticas. Para conocer las imágenes y definiciones, se aplicó el modelo de Tall y Vinner. Encontramos una amplia variedad en las definiciones personales de ángulo de los estudiantes, que no son una memorización de las definiciones dadas en los cursos o en los libros de texto. Además, no poseen una única imagen del concepto de ángulo y su medida, pues evocan diferentes imágenes según el problema a resolver.
\end{abstract}

Fecha de recepción: 04 de septiembre de 2018. Fecha de aceptación: 27 de diciembre de 2019

1 Departamento de Matemática Educativa-Cinvestav IPN, ypachucaherrera@yahoo.com, orcid.org/00000002-8018-2443

2 Departamento de Matemática Educativa-Cinvestav IPN, gzubieta@cinvestav.mx, orcid.org/0000-00022808-6585 
Palabras clave: ángulo; medida de ángulo; definición del concepto; imagen del concepto; nivel superior.

\begin{abstract}
The title of this paper causes a priori bewilderment, because the reader might consider that the concept of angle and its measurement is understood by students starting a bachelor's degree; however, although some difficulties reported in investigations has been overcome, others still persist. In mathematics, the concept definition is relevant, however, in practice, students usually resort to the evoked concept image, which often makes it difficult for them to perform a specific task. A questionnaire was designed based on the notion of angle and its measurement. These questions were answered by 22 Mexican undergraduate students. To identify students' images and definitions, the Tall and Vinner's model was applied. We found a wide variety in the students' personal definitions of angle, which are not memorization of definitions given in their courses or textbooks. In addition, they do not have a single image concept of angle and its measurement, because they evoked different images according to the problem to be solved.
\end{abstract}

Keywords: angle; angle measurement; concept definition; concept image; undergraduate level.

\title{
INTRODUCCIÓN
}

El ángulo y su medida forman parte del estudio de la geometría y la trigonometría. El dominio de estos conceptos es esencial en el aprendizaje de otros cursos de niveles avanzados como: Cálculo, Geometría Analítica, Mecánica, Estática y otros. Sin embargo, como resultado de una investigación previa llevada a cabo con profesores de la educación media superior y alumnos del nivel superior, encontramos la falta de un entendimiento sólido del concepto de medida de ángulo, al mostrar dificultades para interpretar el argumento de la función trigonométrica seno, cuando la unidad de medida es un número real. Pero ¿̇cuál es el origen de estas dificultades? ¿El concepto de ángulo? ¿La medida del ángulo? Estas son algunas de las preguntas que estamos valorando y necesitamos responder. 
Si tomamos en cuenta las investigaciones donde se reporta que, desde la enseñanza elemental, los estudiantes muestran dificultades en el aprendizaje del ángulo y su medida (White y Mitchelmore, 2003; Browning, Garza-Kling y Sundling, 2007; Keiser, 2004), y puesto que existen pocas investigaciones relacionadas con estos conceptos en la enseñanza superior, donde se esperaría encontrar menos dificultades, hemos considerado importante llevar a cabo el siguiente estudio donde nuestro objetivo es conocer la definición e imagen del concepto de ángulo y su medida en estudiantes que inician la educación superior. Los resultados de esta investigación nos permitirán tener un acercamiento al conocimiento real de los estudiantes sobre estos conceptos al iniciar su formación en cursos avanzados de Matemáticas o Física; saber si las dificultades reportadas en investigaciones realizadas en los niveles elementales persisten en los niveles avanzados y, por último, proporcionar a los profesores de dichos cursos elementos para el diseño de actividades que permitan al estudiante tener un mejor desempeño.

\section{ANTECEDENTES}

\section{DEFINICIONES DE ÁNGULO ENCONTRADAS A LO LARGO DE LA HISTORIA}

El concepto de ángulo, esencial en la teoría matemática, fue concebido desde perspectivas diferentes por matemáticos y filósofos. En la obra de Heath (1956), volumen I, hay una recopilación histórica de las diferentes definiciones dadas sobre el ángulo, al tomar como punto de partida las definiciones 8 y 9 de Euclides. Estas definiciones son las siguientes:

8. Un ángulo plano es la inclinación mutua de dos líneas que se encuentran una a otra en un plano y no están en línea recta.

9. Cuando las líneas que comprenden el ángulo son rectas, el ángulo se llama rectilíneo (Heath, 1956, p. 176, nuestra traducción).

Hay otra reseña histórica del concepto de ángulo en los artículos de Matos (1990; 1991) cuyo objetivo es comprender las formas en que los matemáticos entendieron el ángulo, las propiedades que le atribuyeron al concepto y los problemas que resolvieron o no al utilizarlo. Matos (1990) plantea que surgieron debates sobre la naturaleza del ángulo al tratar de responder la pregunta ¿̇a qué categoría aristotélica de cualidad, cantidad o relación pertenece un ángulo de 
acuerdo a su definición? En Matos (1991) continúa la discusión sobre la noción de ángulo, cita la definición dada por Hilbert y expresa que ésta es aceptada por muchos matemáticos contemporáneos y utilizada en las matemáticas escolares en algunos países. Hilbert (1971) definió el ángulo de la siguiente manera:

Sea $\alpha$ un plano cualquiera, $\mathrm{y} h \mathrm{k}$ dos semirrayos diferentes que parten de un punto $O$ en $\alpha$ y que pertenecen a rectas distintas. El sistema de estos dos semirrayos $h, k$ se llama ángulo y lo designaremos con Ł(h,k) o con Ł(k, h) (Hilbert, 1971, p. 11, nuestra traducción).

En sus conclusiones, Matos (1991) señala que la discusión sobre la naturaleza del ángulo aún no ha terminado y que no solo fue abordada por matemáticos, sino también por físicos quienes estaban interesados en cuestiones relativas a la medida del ángulo. Menciona también que los ángulos están asociados con las rotaciones y con las medidas de eventos periódicos, e indica un concepto innovador de ángulo: el ángulo como cantidad de giro.

Basta con observar las obras de Heath (1956) y Matos (1990; 1991) para constatar la transformación de la definición de ángulo con el paso del tiempo, y darse cuenta de cómo cada definición se creó para resolver los problemas de acuerdo a las necesidades en el campo que estudiaba su autor.

\section{DEFINICIONES DE ÁNGULO EN ALGUNOS TEXTOS ESCOLARES SUGERIDOS COMO LIBROS DE CONSULTA}

En los cursos de geometría elemental, se define el ángulo en forma conveniente para el estudio de los triángulos, los criterios de congruencia, semejanza, paralelismo, etcétera. Esta forma se muestra en las siguientes definiciones de dos libros de geometría consultados en el bachillerato. En Moise y Downs (1986) el ángulo se define como sigue: "Si dos rayos tienen el mismo origen o extremo, pero no están en la misma recta, entonces su reunión es un ángulo. Los dos rayos se llaman los lados del ángulo y el extremo común se llama el vértice". (Moise y Downs, 1986, p. 75). Esta definición excluye los ángulos de $0^{\circ}, 180^{\circ}$ y $360^{\circ}$, y los ángulos de más de una vuelta y negativos. Para ilustrarla, añaden las siguientes figuras, que bastan para una adecuada definición en el contexto geométrico (figura 1). 
Un ángulo es una figura como una de éstas:
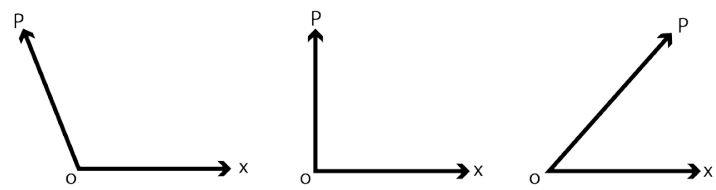

Figura 1. Ángulo definido como pares de semirrectas con un origen común (Moise y Downs, 1986, p. 75).

Mientras en Baldor (1983) el ángulo se define así: "Ángulo es la abertura formada por dos semirrectas con un mismo origen llamado 'vértice'. Las semirrectas se llaman 'lados' [...]". (Baldor, 1983, p. 22). Esta concepción excluye ángulos de $0^{\circ}$, ángulos de más de una vuelta y ángulos negativos. Esta definición la ilustra con las figuras siguientes (figura 2).
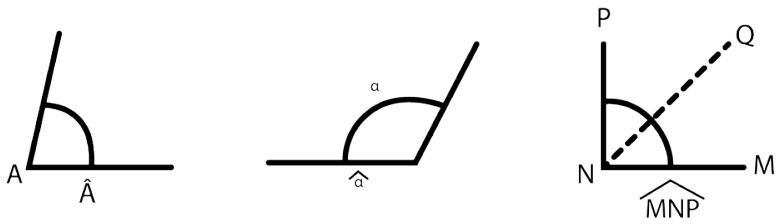

Figura 2. Ángulo definido como abertura (Baldor, 1983, p. 22).

Observemos que, en las ilustraciones de estos dos libros, todos los ángulos tienen un rayo horizontal respecto a la página. Además, en las figuras de ángulo de la primera definición no hay un arco a diferencia de las figuras en la segunda definición. En Moise y Downs (1986) es hasta después de definir la medida de un ángulo que los autores indican con un arco el ángulo del cual se desea obtener su medida, seguramente para evitar la ambigüedad de no saber si se refieren al ángulo convexo o cóncavo.

En textos de cálculo consultados en la educación superior como Spivak (1993), Ayres y Moyer (1991) y Leithold (1992) aparece la definición de ángulo como preámbulo al estudio de las funciones trigonométricas; sin embargo, esta definición aparece de forma diferente a la dada en geometría, pues ésta se amplía y ahora los ángulos se definirán a través de ángulos dirigidos u orientados. Por ejemplo, en Spivak (1993) aparece lo siguiente: 
En geometría elemental, un ángulo es sencillamente la unión de dos semirrectas con un punto común inicial [...] Más útiles para la trigonometría son los 'ángulos dirigidos', los cuales pueden ser considerados como pares $\left(1_{1}, 1_{2}\right)$ de semirrectas con el mismo punto inicial (Spivak, 1993, pp. 425-426).

Spivak añade la siguiente figura (figura 3).
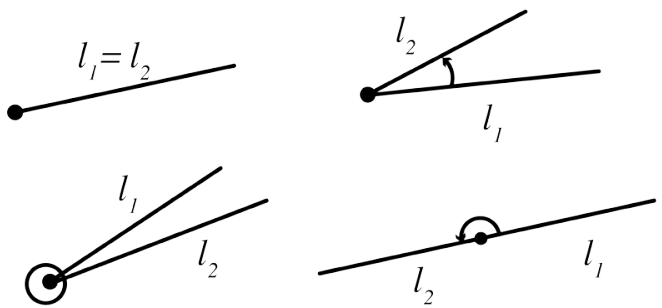

Figura 3. Ángulos dirigidos (Spivak, 1993, p. 426.)

Ayres y Moyer (1991) mencionan lo siguiente respecto al ángulo plano:

Más comúnmente, puede pensarse que un ángulo plano se genera si se gira (en un plano) una línea de la posición inicial $O X$ a la posición terminal $O P$. Entonces, $O$ es otra vez el vértice, $\overrightarrow{O X}$ al que se llama lado inicial, y $\overrightarrow{O P}$ se llama lado terminal del ángulo (Ayres y Moyer, 1991, p. 1).

Con la figura siguiente ilustran su definición (figura 4).
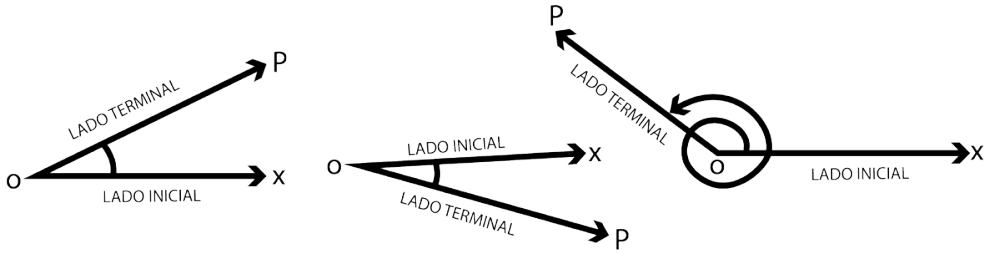

Figura 4. Ángulos planos generados a través de giros (Ayres y Moyer, 1991, p. 2). 
Leithold (1992) escribe lo siguiente:

Cualquier ángulo es congruente con el ángulo que tenga su vértice en el origen y un lado, llamado lado inicial, sobre el lado positivo del eje $x$. Un ángulo tal se dice que está en posición normal. La Figura 5 muestra un ángulo AOB en posición normal con $\mathrm{OA}$ como lado inicial. El otro lado, $\mathrm{OB}$, se llama lado terminal. El ángulo $\mathrm{AOB}$ puede ser formado girando el lado OA hasta el lado OB [...] (Leithold, 1992, p. 61)

El autor ilustra la definición con la figura siguiente, que incluye ejes cartesianos (figura 5).

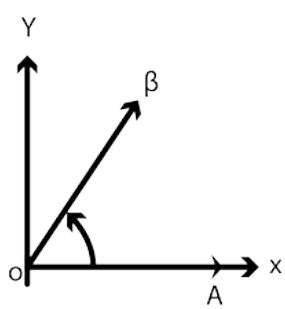

Figura 5. Ángulo en posición normal (Leithold, 1992, p. 61).

Estas tres definiciones incluyen ángulos positivos, negativos, de $0^{\circ}, 180^{\circ}, 360^{\circ} \mathrm{y}$ ángulos de más de una vuelta. Cabe destacar que en las ilustraciones de los ángulos de las figuras 4 y 5 todas tienen un lado alineado con la horizontal. Mientras que solo en la figura 4 se representan ángulos negativos y de más de una vuelta. Todas estas definiciones (las dadas en geometría y en trigonometría) descartan la posibilidad de que al menos uno de los lados del ángulo pueda ser una línea curva.

En resumen, encontramos distintas definiciones de ángulo tanto en geometría como en trigonometría, esta diversidad tiene que ver con el objetivo que pretende cada autor, y está ajustada a la estructura matemática de la que se está haciendo uso.

\section{MEDIDA ANGULAR}

Haremos referencia a Moise y Downs (1986), quienes escriben: "Así como medimos segmentos con una regla, medimos ángulos con un transportador". En seguida agregan lo siguiente: "El número de grados de un ángulo se llama su 
medida. Si hay $r$ grados en el Ł PQR, entonces escribimos mъ $\mathrm{PQR}=r^{\prime \prime}$. (Moise y Downs, 1986, p. 81).

Por otra parte Baldor (1983), en la sección titulada medida de ángulos señala que: "Medir un ángulo es compararlo con otro que se toma por unidad" (Baldor, 1983, p. 23).

\section{LA MEDIDA ANGULAR EN LA PRÁCTICA}

En la escuela, la medida angular se obtiene usualmente utilizando al menos uno de los siguientes recursos: comparación con ángulos de referencia, uso del transportador y el uso de postulados y definiciones. A continuación describimos estos recursos.

Comparación con ángulos de referencia. Se puede aproximar la medida de un ángulo al tomar como referencia ángulos cuya medida es conocida, por ejemplo, el ángulo recto cuya medida es de $90^{\circ}$, el llano que mide $180^{\circ}$, el ángulo agudo (mide menos de $90^{\circ}$ ), entre otros. Por ejemplo, se puede saber una medida aproximada del ángulo de la figura 6 , si tomamos como referencia el ángulo recto, entonces la medida del ángulo sería aproximadamente la mitad de la unidad que es el ángulo recto.

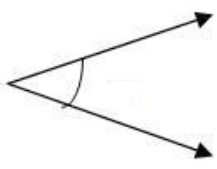

Figura 6. Ejemplo de un ángulo del cual se desea conocer su medida.

Uso del transportador. Para obtener la medida de un ángulo podemos utilizar como herramienta el transportador. Esta herramienta utiliza el grado como unidad de medida.

Uso de postulados y otras definiciones. Para determinar la medida de ángulos pueden usarse postulados y otras definiciones como: el postulado de la adición de ángulos, la definición de par lineal, de ángulos suplementarios, entre otros. 


\section{INVESTIGACIONES RELACIONADAS CON LA ENSEÑANZA Y EL APRENDIZAJE DEL ÁNGULO Y SU MEDIDA}

Varias investigaciones reportan que, desde la enseñanza elemental, los estudiantes muestran dificultades en el aprendizaje del concepto de ángulo, como las de White y Mitchelmore (2003), Browning y Garza-Kling y Sundling (2007) quienes se enfocaron en la formación del concepto de ángulo a partir de experiencias físicas. Otra, es el trabajo de Keiser (2004), quien hizo una comparación entre la evolución que ha tenido el concepto de ángulo a través de la historia, y las concepciones del estudiante en tres direcciones: en cuanto a la medida del ángulo, si los ángulos pueden incluir curvas y las dificultades en conceptualizar ángulos de $0^{\circ}, 180^{\circ}$ y $360^{\circ}$. Todos estos trabajos se llevaron a cabo con estudiantes de niveles de educación básica.

Algunos de los resultados encontrados en estas investigaciones, como los de White y Mitchelmore (2003), señalan que el concepto de ángulo es un concepto multifacético difícil de aprender, al encontrar en los estudiantes dificultades al identificar los lados que forman un ángulo, por ejemplo, el que se forma al abrir una puerta al no haber un lado visible o con los ángulos de inclinación debido a que no están familiarizados con la idea del ángulo que se forma con la horizontal. Además, mencionan que les fue difícil determinar lo que los estudiantes intentaban comunicar cuando se les pidió que definieran el concepto de ángulo.

Mientras, Browning, Garza-Kling y Sundling (2007) mencionan que los estudiantes tienen nociones de ángulo tan limitadas que tienen dificultades en proporcionar una definición matemática adecuada para el término y, en cambio, describen lo que estamos midiendo cuando medimos un ángulo, por ejemplo, cuando mencionan que un ángulo es la "cantidad de grados" o "el número de grados". Además, con frecuencia no reconocen que dos ángulos tienen la misma medida si están orientados en direcciones no estándar, y agregan que algunos adultos aún luchan con la identificación de ángulos de 90 grados que no tienen un rayo horizontal.

Entre otras dificultades, los estudiantes tienden a atribuir la medida de un ángulo a la longitud de los segmentos de recta que lo constituyen, algunos consideran que en el dibujo de las letras $\mathrm{R}$ o $\mathrm{P}$ puede haber ángulos al mirar intersecciones entre líneas y curvas, es decir, ellos observan dos líneas que se "conectan" en un punto; en cambio en el dibujo de la letra S no puede haber ángulos porque no observan intersecciones en ningún lado, puesto que 
mencionan "es un solo trazo". No conciben un ángulo de 180 grados porque no ven dos líneas que se conectan y el ángulo de 360 grados no lo consideran un ángulo al no visualizar dos lados que se unen "en una esquina". También es probable que los estudiantes de los niveles básicos carezcan de un vocabulario adecuado para formular descripciones claras del ángulo (Keiser, 2004).

En cuanto a la medida angular, Pachuca (2014) reporta que profesores de la educación media superior y alumnos del nivel superior tienen dificultades para interpretar el argumento de la función trigonométrica seno, cuando la unidad de medida es un número real. En las respuestas, cuando se les pide evaluar la función seno en un número real cualquiera, eligieron la medida angular al relacionar el valor del argumento con algún símbolo, número, o expresión matemática conocido por ellos, por ejemplo, cuando el valor del argumento era 180 lo consideraron como grados al asociar el número 180 con $180^{\circ}$ aun cuando el número 180 no tenía el símbolo de grados o simplemente le agregaron el símbolo de grados. Cuando en el argumento aparecía el valor $\pi$, por ejemplo, en 180/ $\pi$ consideraron como medida angular los radianes porque asociaron el número $\pi$ con radianes. Para números como -7, o 0.357 algunos no sabían interpretar el argumento. Mientras, Akkoc (2008) reporta que profesores en formación tuvieron dificultades con el concepto de radián, además de evidenciar que la imagen del concepto de grado predomina sobre la de radián concluyendo que estas dificultades podría causar problemas para comprender las funciones trigonoméricas.

\section{MARCO TEÓRICO}

Entre los enfoques que estudian la formación de conceptos, tenemos el desarrollado por Tall y Vinner (1981) quienes confrontan los conceptos matemáticos como se definen formalmente y los procesos cognitivos mediante los cuales son concebidos. Estas ideas se formalizaron a través de la llamada imagen del concepto y la definición del concepto.

De acuerdo a Vinner (2011) el concepto es una idea evocada en nuestra mente cuando escuchamos o vemos el nombre del concepto. El concepto existe solo en nuestra mente y puede ser considerado algo abstracto. Por su parte, el nombre del concepto es una entidad lingüística, usualmente llamada una noción o un término que tiene un aspecto físico (visual o vocal). Según Vinner (2002), cuando vemos o escuchamos el nombre de un concepto, algo es evocado en 
nuestra memoria, y lo evocado usualmente no es la definición del concepto sino lo que él y Tall denominaron "imagen del concepto". Esta imagen es algo no verbal asociado en nuestra mente con el nombre del concepto, puede ser una representación visual del concepto cuando tenga representaciones visuales, imágenes mentales, una colección de impresiones o experiencias, y estas pueden traducirse a formas verbales.

En el caso del concepto de ángulo, la imagen del concepto que se crea en la mente de los estudiantes está compuesta por las diferentes representaciones que recuerdan relacionadas con dicho concepto, junto con las distintas propiedades que el estudiante le asocia. Esta imagen es personal, puede variar de persona a persona o de una cultura a otra, por lo tanto, sólo es posible hablar de una imagen conceptual en relación con un individuo en particular.

Tall y Vinner (1981), también introducen el término "imagen evocada del concepto" para describir la parte de la memoria evocada en un tiempo dado o en un contexto dado, es decir, aquella porción de la imagen del concepto activada en una situación particular, lo cual no es necesariamente todo lo que un cierto individuo sabe sobre una noción particular.

Para Tall y Vinner (1981) la "definición del concepto" se entiende como una definición verbal que explica con precisión el concepto. La definición del concepto abarca desde la definición formal, es decir, la aceptada por la comunidad científica en su conjunto, hasta la definición personal del concepto, la cual se usa para expresar con nuestras propias palabras la definición de un concepto. La definición personal puede diferir de la formal y se utiliza para construir o reconstruir la definición formal del concepto.

Vinner (2002) dice que al plantear una tarea cognitiva al alumno, al parecer algunos profesores esperan que en los procesos intelectuales relacionados con la realización de una tarea determinada, los estudiantes basen sus razonamientos principalmente en las definiciones formales del concepto mientras sus imágenes del concepto juegan un papel secundario. Vinner y Hershkowitz (1980) representan esto con el diagrama de la figura 7, donde las flechas indican diferentes formas de funcionamiento de un sistema cognitivo. 


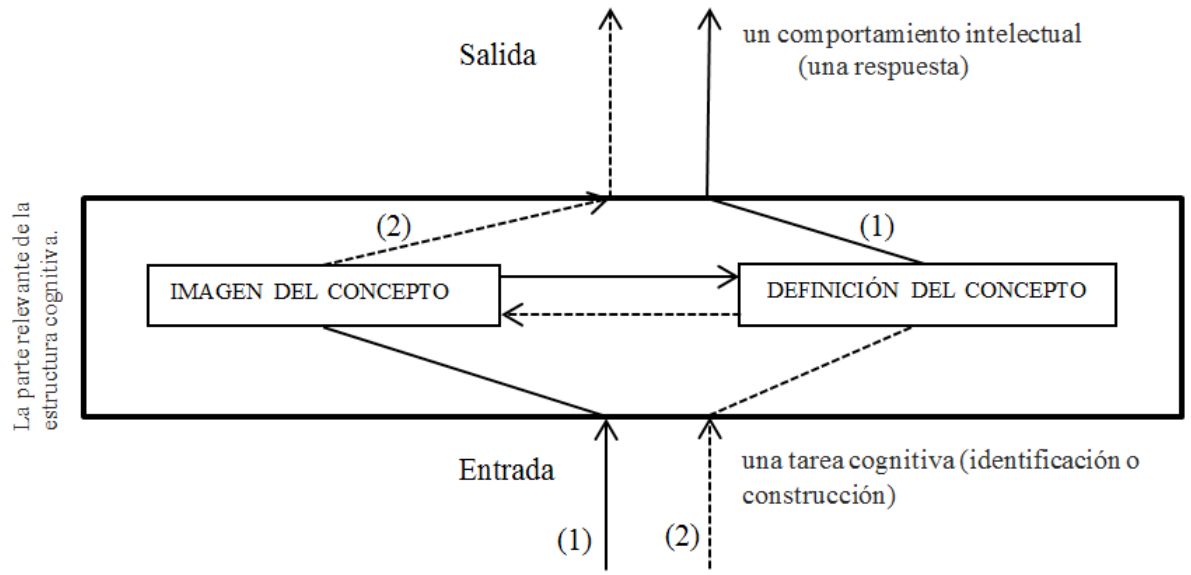

Figura 7. Modelo de actividad mental de los estudiantes esperado por los profesores, adaptado de Vinner y Hershkowitz (1980).

Pero, desafortunadamente, la práctica es diferente. Aquí, explica Vinner (2002), la celda de definición del concepto, incluso si no es nula, no se consulta durante el proceso de resolución de problemas. Los hábitos de pensamiento de la vida cotidiana toman el control, y el encuestado no está consciente de la necesidad de consultar la definición formal. Por lo tanto, un modelo más apropiado, para los procesos que ocurren en la práctica, es el siguiente (ver la figura 8):

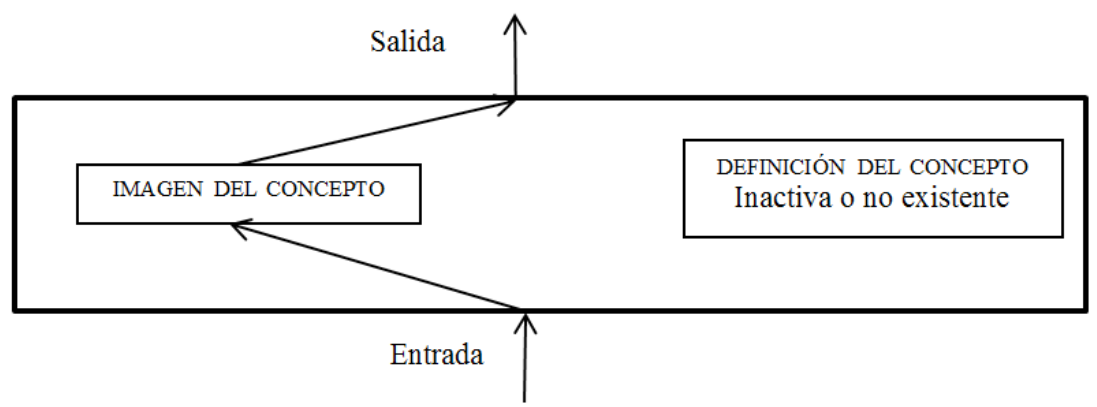

Figura 8. Modelo real de actividad mental de muchos estudiantes, adaptado de Vinner y Hershkowitz (1980). 
Vinner (2002) agrega que el aprendizaje de las matemáticas impone a los estudiantes ciertas maneras de pensar distintas al aprendizaje cotidiano, pues las definiciones juegan un rol fundamental, y se espera que los estudiantes entiendan que el significado de un concepto está determinado por su definición formal y no por sus puntos de vista previos sobre el concepto. Este sería el proceso deseable, sin embargo, si se toma en cuenta que la mente de los estudiantes tiende a basarse en su imagen del concepto y no en su definición, puesto que esta definición se vuelve inactiva por ser difícil de entender, porque nunca la aprendieron, porque se olvida o se ignora, entonces se debería hacer un esfuerzo por formar imágenes correctas en la mente del estudiante. De hecho, Vinner y Hershkowitz (1980) muestran los resultados de una prueba aplicada a estudiantes de grados 7,8 y 9 relacionados con figuras geométricas como el ángulo. Encontraron que los ángulos obtusos con un rayo horizontal son identificados más fácilmente que otros ángulos obtusos colocados en una posición distinta. De este resultado comentan que los profesores y los libros de texto tienden a dibujar ángulos con un rayo horizontal, y como resultado de este hecho la imagen del concepto puede contener ángulos obtusos con un rayo horizontal.

Finalmente, Vinner (2002) señala que se deben proponer ejemplos o tareas que permitan al estudiante ver los conflictos que pueden surgir entre la imagen del concepto que posee y la definición formal del concepto, discutir esos conflictos con la intención de convencer al estudiante sobre el rol tan importante que juegan las definiciones en los contextos técnicos, particularmente en matemáticas. Es decir, solo si se le plantean tareas que no pueda resolver correctamente refiriéndose solo a su imagen del concepto, se le podría convencer de lo conveniente que es utilizar la definición del concepto.

\section{PREGUNTAS DE INVESTIGACIÓN}

Para este estudio se formularon las siguientes preguntas de investigación que nos sirvieron de guía:

- ¿Qué definiciones tienen los estudiantes del ángulo al iniciar la enseñanza superior?

- ¿Qué imágenes evocan los estudiantes del ángulo y su medida, al contestar ítems relacionados con estos conceptos? 


\section{MÉTODO}

El método de nuestro estudio es cualitativo. Este tipo de método es particularmente apropiado cuando el propósito es examinar las "formas de expresión" propias a cada individuo, es decir, los conceptos, percepciones, imágenes mentales, etc, manifestadas en el lenguaje de los participantes, ya sea de manera individual, grupal o colectiva (Sampieri, 2018). Investigamos las imágenes y definiciones del concepto de ángulo y su medida en estudiantes universitarios. Como técnicas de recolección de datos, utilizamos un cuestionario y entrevistas semiestructuradas individuales. La muestra elegida para aplicar el cuestonario se obtuvo a través de un muestreo por conveniencia. Participaron un total de 22 estudiantes de primer semestre de la Licenciatura en Física y Matemáticas (17 a 19 años) de una universidad pública de la Ciudad de México. Su preparación era variada puesto que provenían de escuelas del nivel medio superior públicas y privadas, de la Ciudad de México y de otros estados de la República Mexicana. Consideramos estudiantes de esta licenciatura puesto que en los hechos, en el área de las ciencias exactas se encuentran, en promedio, los estudiantes con mayor dominio de las matemáticas.

Iniciamos nuestra investigación aplicando un cuestionario escrito de 12 ítems relacionados con el ángulo y su medida. En su diseño se tomaron en cuenta las dificultades reportadas en investigaciones previas, se revisaron los planes de estudio desde los niveles básicos hasta el bachillerato, con el fin de identificar los aspectos clave que el estudiante debe poseer al iniciar el primer semestre de su carrera. Los aspectos a considerar son: definición de ángulo, representación gráfica de un ángulo, distintos tipos de ángulos y medida de un ángulo. Su elaboración también se basó en Vinner (2002) quien señala que un método natural para aprender acerca de la definición del concepto de un individuo es a través de preguntas directas, como por ejemplo, ¿qué es una función?, puesto que las definiciones son verbales y explícitas, mientras que para aprender acerca de la imagen del concepto, se deben plantear preguntas indirectas puesto que la imagen del concepto puede ser verbal e implícita.

En este artículo presentamos el análisis de las respuestas a cuatro ítems, mostrados en el anexo. Esta selección se basó en el hecho de que en los ítems restantes, los estudiantes no mostraron dificultades en sus respuestas, o bien no aportaron información relevante adicional para este estudio. El ítem 1 nos ayudará a responder la primera pregunta de investigación: ¿qué definiciones tienen los estudiantes del ángulo al iniciar la enseñanza superior?, y los ítems 2, 4 y 10 para responder la 
segunda pregunta de investigación: ¿qué imágenes evocan los estudiantes del ángulo y su medida, al contestar ítems relacionados con estos conceptos?

Los estudiantes tuvieron 45 minutos para responder el cuestionario. Se les comentó que su participación era voluntaria y que sus respuestas serían anónimas y confidenciales. A continuación, indicamos en la tabla 1 los propósitos de cada uno de los cuatro ítems.

Tabla 1. Descripción de los ítems

\begin{tabular}{|c|c|c|c|}
\hline Ítem & Objetivo & Intención particular & Fundamentación \\
\hline $\begin{array}{l}\text { Escribe la defini- } \\
\text { ción de ángulo }\end{array}$ & $\begin{array}{l}\text { Conocer la definición } \\
\text { de ángulo que posee } \\
\text { el estudiante }\end{array}$ & $\begin{array}{l}\text { Saber si los estudiantes } \\
\text { continúan definiendo el } \\
\text { ángulo a través de su } \\
\text { medida }\end{array}$ & $\begin{array}{l}\text { Libros de texto reportados en } \\
\text { los antecedentes. } \\
\text { De acuerdo con Browning, } \\
\text { Garza-Kling y Sundling (2007), } \\
\text { algunos estudiantes de nivel } \\
\text { básico definen el ángulo a tra- } \\
\text { vés de su medida }\end{array}$ \\
\hline $\begin{array}{l}\text { Dibuja un ángulo } \\
\text { y señala cada una } \\
\text { de sus partes }\end{array}$ & $\begin{array}{l}\text { Conocer la imagen } \\
\text { evocada del estudian- } \\
\text { tes al hacer la repre- } \\
\text { sentación gráfica del } \\
\text { ángulo }\end{array}$ & $\begin{array}{l}\text { Saber si la imagen del } \\
\text { concepto que evoca el } \\
\text { estudiante está relacio- } \\
\text { nada con ángulos en } \\
\text { posición no estándar }\end{array}$ & $\begin{array}{l}\text { En Vinner y Hershkowitz } \\
\text { (1980) se señala que los profe- } \\
\text { sores y los libros de texto tien- } \\
\text { den a dibujar ángulos con un } \\
\text { rayo horizontal, y como resul- } \\
\text { tado de este hecho la imagen } \\
\text { puede contener ángulos con } \\
\text { un rayo horizontal }\end{array}$ \\
\hline $\begin{array}{l}\text { Determina la o las } \\
\text { figuras que consi- } \\
\text { deres ángulos. (Tá- } \\
\text { chalos) }\end{array}$ & $\begin{array}{l}\text { Conocer qué imagen } \\
\text { evoca el estudiante } \\
\text { para decidir si una fi- } \\
\text { gura dada es un án- } \\
\text { gulo o no }\end{array}$ & $\begin{array}{l}\text { Saber si los estudiantes } \\
\text { consideran ángulos en- } \\
\text { tre líneas y curvas. } \\
\text { Saber si los estudiantes } \\
\text { pueden identificar án- } \\
\text { gulos de } 180^{\circ}\end{array}$ & $\begin{array}{l}\text { De acuerdo con Keiser (2004), } \\
\text { algunos estudiantes de nivel } \\
\text { básico consideran que puede } \\
\text { haber ángulos entre líneas y } \\
\text { curvas, además se les dificul- } \\
\text { ta identificar ángulos de } 180^{\circ}\end{array}$ \\
\hline $\begin{array}{l}\text { Relaciona cada } \\
\text { ángulo con su me- } \\
\text { dida. (Puedes rela- } \\
\text { cionar las figuras } \\
\text { con más de una } \\
\text { opción) }\end{array}$ & $\begin{array}{l}\text { Conocer la imagen } \\
\text { evocada al momento } \\
\text { de relacionar cada án- } \\
\text { gulo con su medida }\end{array}$ & $\begin{array}{l}\text { Saber si los estudiantes } \\
\text { reconoce que dos ángu- } \\
\text { los tienen la misma me- } \\
\text { dida, aunque estén en } \\
\text { diferentes posiciones }\end{array}$ & $\begin{array}{l}\text { Browning, Garza-Kling y } \\
\text { Sundling (2007), reportan que } \\
\text { los estudiantes de niveles bá- } \\
\text { sicos no reconocen que dos } \\
\text { ángulos tienen la misma me- } \\
\text { dida si están orientados en } \\
\text { direcciones no estándar }\end{array}$ \\
\hline
\end{tabular}


Después de un primer análisis de la información recopilada de los cuestionarios -detallado más adelante-, seleccionamos algunos estudiantes para entrevistarlos de forma individual. El propósito de las entrevistas era conocer a partir del lenguaje y perspectiva del estudiante, "en sus propias palabras", su imagen evocada. La selección se hizo con base en las respuestas que consideramos proporcionaban información relevante. Se les informó que la intención de la entrevista era profundizar en sus respuestas. A las entrevistas se les aplicó un análisis temático o de contenido (Sampieri, 2018), utilizando como referente las preguntas de investigación.

\section{ANÁLISIS DE DATOS Y RESULTADOS}

Ítem 1: Definición de ángulo. A cada una de las respuestas de los estudiantes a este ítem se les hizo una codificación abierta (Sampieri, 2018), utilizando segmentos de texto de sus respuestas. Las categorías que emergieron fueron 6: C1, C2, C3, C4, C5 y C6. A continuación, presentamos cada categoría y su código, señalando algunas respuestas que pertenecen a cada una de ellas. En cada categoría especificamos el número de respuestas, por ejemplo (4/22) indica que 4 de las 22 respuestas pertenecen a esta categoría.

C1: El ángulo definido como apertura/abertura de segmentos (rayos o líneas) (7/22)

Ejemplo 1: "Es la apertura o separación de dos rayos que tienen un punto en común". Ejemplo 2: "Abertura que existe entre dos rayos con un origen en común".

C2: El ángulo definido como intersección de dos rayos (líneas o semiplanos) (4/22)

Ejemplo 1: "Es la intersección de dos líneas diferentes las cuales tienen un punto en común".

Ejemplo 2: "Es la intersección de dos semiplanos formados por dos rayos con un mismo vértice".

C3: El ángulo definido a través de su medida (3/22)

Ejemplo: "Es la medida de la apertura de dos líneas que se unen en un punto". 
C4: El ángulo definido como medida particular (3/22)

Ejemplo: "Es la separación entre dos rectas perpendiculares".

C5: Otras definiciones (2/22)

Ejemplo: "Un ángulo es cuando dos rayos se cruzan entre si".

C6: No respondió/No conoce la definición (3/22)

Ejemplo: “No conozco la definición, porque no tengo una definición muy concreta y me falta más información".

En las respuestas se observa una amplia variedad en las definiciones personales de ángulo de los estudiantes al definirlo como: la intersección de dos rayos/ líneas o semiplanos, la apertura/abertura de dos rayos o líneas, a través de su medida o de una medida en particular.

Ítem 2. Representación gráfica del ángulo. Aquí revisamos las figuras que hicieron los estudiantes para representar un ángulo. Notamos algunas características comunes que predominaban en la mayoría de las figuras. Una primera característica fue la posición del ángulo, puesto que todos los estudiantes dibujaron ángulos colocados de tal manera que su vértice estaba ubicado en el lado izquierdo, uno de sus lados estaba alineado o no con la horizontal, mientras el otro lado se hallaba por encima de éste como lo muestra la figura 9.

\section{Dibuja un ángulo y señala cada una de sus partes.}
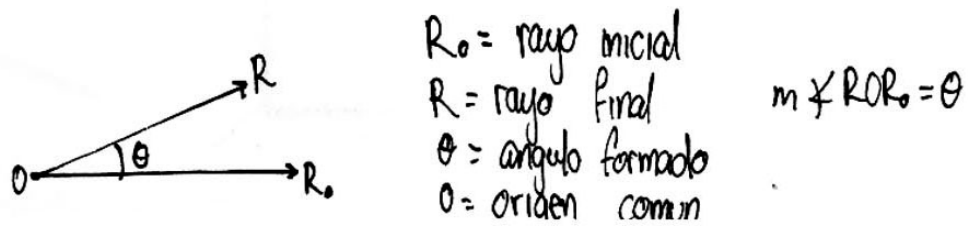

Figura 9. Ejemplo de una representación gráfica de ángulo de uno de los estudiantes, con el vértice ubicado a la izquierda y uno de sus lados alineado con la horizontal.

Otra característica fue que 15 estudiantes trazaron ángulos agudos, con uno de sus lados alineado con la horizontal, de los cuales doce eran como los de la figura 9 , y tres como los de la figura 10, donde no señalan ningún arco. 
2. Dibuja un ángulo y señala cada una de sus partes.
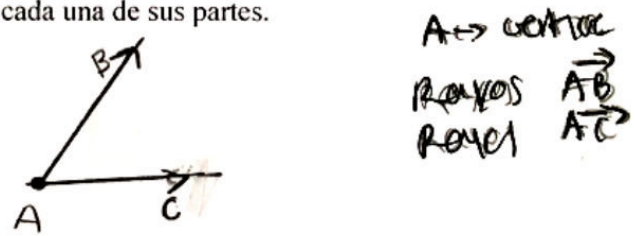

Figura 10. Ejemplo de una representación gráfica de ángulo de uno de los estudiantes, con uno de sus lados alineado con la horizontal donde no señalan ningún arco.

Los ángulos que no tenían un lado horizontal eran ángulos agudos como el de la figura 11.

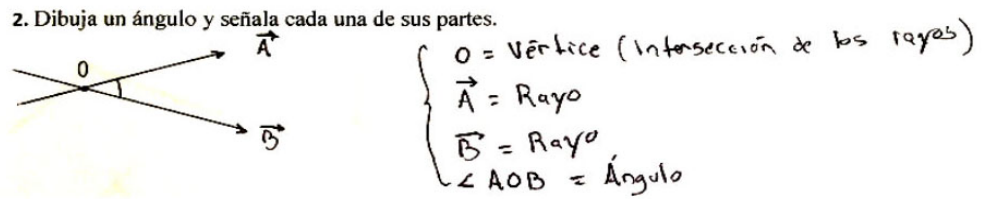

Figura 11. Ejemplo de una representación gráfica de ángulo de uno de los estudiantes, con uno de sus lados no alineado con la horizontal.

18 estudiantes trazaron dos rayos, segmentos o líneas distintas que emanaban de un punto, y en algunos casos nombraron sus partes (véanse figuras 9 y 10), mientras que los otros cuatro dibujaron dos rayos, líneas o lados distintos que se intersecaban, y nombraron sus partes. (véase figura 11).

Ítem 4. Imagen evocada del concepto de ángulo para decidir si una figura es un ángulo o no. En el análisis de las respuestas creamos dos categorías. En la categoría C1 colocamos las figuras que los estudiantes consideraban como ángulos, y en $\mathrm{C} 2$ las que no. Se revisaron las categorías y encontramos que 5 de los 22 estudiantes consideraron la figura 12 un ángulo.

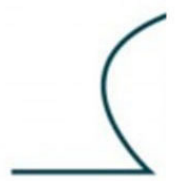

Figura 12. Figura del cuestionario considerada por algunos estudiantes como un ángulo. 
A continuación, veremos un extracto de la entrevista donde el estudiante expresa el porqué determinó que la figura 12 es un ángulo. El estudiante será identificado como estudiante 1.

Entrevistadora: ¿̇Por qué determinaste que esta figura era un ángulo? [se refiere a la figura 12].

Estudiante 1: $\quad$ iAh! bueno, porque pues tenemos que tomar esta línea [señala con su dedo índice la línea horizontal de la figura 12], entonces aquí pues hay otra [señala con su dedo índice la curva en la misma figura] que hasta cierto punto haciendo un acercamiento cada vez más y más grande se podría ver que ya no conectamos en una curva si no que hay un punto en el que va a tener un comportamiento de línea recta. Un acercamiento muy grande como hacerle un super zoom.

La imagen evocada por el estudiante 1 está asociada con una imagen formada por dos líneas rectas diferentes con un punto en común, que forman una cuña o una punta. De los cuatro estudiantes restantes que contestaron de manera similar al estudiante 1, entrevistamos a tres de ellos y nos proporcionaron argumentos similares.

Otro caso de interés es el de un alumno que señaló que la figura 13 no era un ángulo.

Figura 13. Figura del cuestionario que no fue considerada como un ángulo por un estudiante.

Veamos a continuación un extracto de la entrevista que se le realizó a este estudiante, el cuál será identificado como estudiante 2.

Entrevistadora: Esta figura [la figura 13] ¿por qué no la consideraste como ángulo?

Estudiante 2: Es que ese me confundí un poco no sé si sea un ángulo de 0 o de...sí pero no, no creo porque es una línea.

Entrevistadora: $\quad$ Pero esta es también una línea [se le señala la figura 14] ¿por qué esta sí la consideraste un ángulo y esta no? 
Estudiante 2: $\quad$ Bueno lo que yo observé es que aquí está el punto lel alumno coloca su dedo índice sobre la figura 14 del lado derecho], está donde empieza el ángulo [enseguida traza una trayectoria con su dedo índice como se muestra en la figura 15] y esta es la abertura que tiene, en esta de acá [señala la figura 13] no se ve claramente [se refiere a que no se ve abertura de acuerdo a sus palabras].

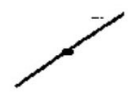

Figura 14. Figura del cuestionario que fue considerada como un ángulo por el estudiante 2.

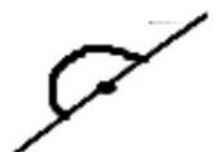

Figura 15. Representación de la trayectoria trazada por el estudiante 2.

Aquí la imagen evocada del estudiante 2 está asociada con dos líneas diferentes con un punto común que forman una "abertura". Dos estudiantes más contestaron de manera similar al estudiante 2, ambos fueron entrevistados proporcionando argumentos similares.

Ítem 10. Imagen evocada al relacionar un ángulo con su medida. En este ítem, los estudiantes tenían que relacionar cuatro ángulos con una columna de respuestas dadas. Dos de los ángulos son los ángulos $\mathrm{A}$ y $\mathrm{B}$ que se muestran en la figura 16. Estos ángulos tienen la misma medida, sin embargo, están colocados en posiciones diferentes.

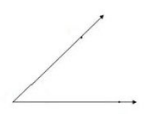

Ángulo A

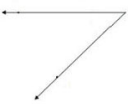

Ángulo B

Figura 16. Ángulos del cuestionario que los estudiantes tenían que relacionar con su medida. 
16 estudiantes relacionaron los dos ángulos A y B con la respuesta a) "Ángulo menor a $90^{\circ}$. Sin embargo, hubo 4 estudiantes que relacionaron el ángulo $\mathrm{A}$ con la respuesta a) "Ángulo menor a $90^{\circ "}$ y el ángulo B con otra respuesta, la b) "Ángulo mayor a $180^{\circ}$ ". A continuación presentamos un extracto de la entrevista realizada a uno de estos cuatro estudiantes sobre la medida que asignó al ángulo B. Este estudiante será identificado como estudiante 3.

Entrevistadora: En este ítem te voy a preguntar por esta figura [señala el ángulo B de la figura 16], me dices que lo relacionas con la respuesta b), "Ángulo mayor a 180 grados", si me puedes explicar, ¿̇por qué lo relacionaste con esta opción?

Estudiante 3: $\quad$ Pues igual que el anterior, por lo mismo de que me imaginé la línea prolongada [coloca su dedo índice en el vértice del ángulo dado y con este traza una prolongación del segmento horizontal efectuando el movimiento de izquierda a derecha. Véase la línea punteada de la figura 17] y tomé como este el eje $x$ para poder hacer el ángulo [coloca su dedo índice sobre la prolongación que trazó, y considerándola como lado inicial, dibuja una trayectoria como se muestra en la figura 17 hasta llegar al segmento inclinado].

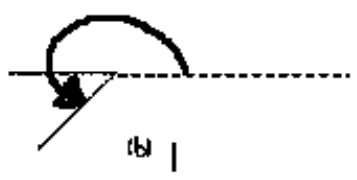

Figura 17. Ilustración del razonamiento considerado por el estudiante 3 para determinar que la medida del ángulo B es mayor a 180 grados.

La imagen evocada en este caso está relacionada con ángulos dirigidos. Aquí el alumno ignoró el segmento horizontal dado como uno de los lados del ángulo B. Trazó imaginariamente una prolongación del segmento horizontal, el cual tomó como lado inicial y como lado final el inclinado. De los tres estudiantes restantes que contestaron de manera similar al estudiante 3, entrevistamos a dos de ellos y nos proporcionaron argumentos similares.

Ahora, veamos las respuestas asociadas a los dos ángulos restantes del ítem 10. (véase tabla 2). Si observamos los ángulos $C$ y D estos son el mismo (ambos tienen la misma medida: $0^{\circ}$ ), salvo que el ángulo $\mathrm{D}$ es el ángulo $\mathrm{C}$ pero rotado 180 grados. 
Tabla 2. Respuestas de los estudiantes

\begin{tabular}{ccc|ccc}
\hline Ángulo C & $\begin{array}{c}\text { Número de } \\
\text { estudiantes }\end{array}$ & $\begin{array}{c}\text { Medida } \\
\text { relacionada }\end{array}$ & Ángulo D & $\begin{array}{r}\text { Número de } \\
\text { estudiantes }\end{array}$ & $\begin{array}{c}\text { Medida } \\
\text { relacionada }\end{array}$ \\
\hline 5 & $0^{\circ}$ & 4 & $0^{\circ}$ \\
11 & $0^{\circ}, 360^{\circ}$ & 5 & $0^{\circ}, 360^{\circ}$ \\
1 & $0^{\circ}, 180^{\circ}$ & - & 2 & $0^{\circ}, 180^{\circ}$ \\
2 & $0^{\circ}, 180^{\circ}, 360^{\circ}$ & & $0^{\circ}, 180^{\circ}, 360^{\circ}$ \\
2 & $180^{\circ}$ & & 1 & $180^{\circ}$ \\
1 & Sin respuesta & Sin respuesta \\
\hline
\end{tabular}

En la tabla 2 observamos, que 8 estudiantes asignaron la medida de $180^{\circ}$ al ángulo D. A continuación mostramos un extracto de la entrevista realizada a uno de ellos, el cual será identificado como estudiante 4. Este estudiante inicialmente asignó al ángulo $\mathrm{C}$ la respuesta d) "Ángulo igual a 0", sin embargo, al ángulo D, le asignó las respuestas f) "Ángulo igual a $180^{\circ "}$ y c) "Ángulo mayor a $90^{\circ}$ ". En la entrevista, el estudiante menciona por qué asignó esas opciones.

Entrevistadora: Vamos a la siguiente figura [ángulo D], esa tú la relacionaste con f), “Ángulo igual a $180^{\circ \prime}$ y c), "Ángulo mayor a $90^{\circ \prime}$. Me podrías explicar, ¿ipor qué lo relacionaste así?

Estudiante 4: $\quad$ Igual, me imaginé la línea prolongada y lo hice lel alumno coloca su dedo índice en el vértice del ángulo dado y con este traza una prolongación del segmento horizontal efectuando el movimiento de izquierda a derecha. Véase la línea punteada de la figura 18. Después coloca su dedo índice sobre la prolongación que trazó y considerándola como lado inicial dibuja una trayectoría como se muestra en la figura 18 hasta llegar al segmento dado].

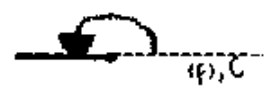

Figura 18. Representación de la trayectoria trazada por el estudiante 4. 
En este caso, para el estudiante un ángulo se forma con dos segmentos diferentes. Pero en la figura él sólo ve un segmento, razón por la cual traza imaginariamente otro, el cual de acuerdo al movimiento de su dedo índice, en una rotación, lo considera como el lado inicial, y el dado en la figura como lado final de un ángulo dirigido de 180 grados. A los siete estudiantes restantes que contestaron que la medida del ángulo D es de $180^{\circ}$, se les hizo una entrevista proporcionando argumentos similares a los del estudiante 4. Así concluimos nuestro análisis.

\section{DISCUSIÓN DE RESULTADOS}

La descripción de las respuestas nos permitió conocer las diferentes definiciones e imágenes evocadas por los estudiantes al contestar ítems relacionados con el ángulo y su medida. Vamos a centrar la discusión en los aspectos más relevantes extraídos de los resultados obtenidos.

Ítem 1. Conocer la definición de ángulo que posee el estudiante. La mayoría de las definiciones están en la categoría C1, (apertura/abertura de segmentos, rayos o líneas), además de que las pertenecientes a esta categoría (las que están en términos de abertura) son similares a la definición dada en Baldor (1983). Seis estudiantes (C3 y C4) definieron el ángulo a través de su medida o como una medida en particular, esto indica que en los niveles superiores los estudiantes aún tienen dificultades en distinguir entre el concepto de ángulo y su medida, como viene sucediendo desde los niveles básicos, de acuerdo con Browning, Garza-Kling y Sundling (2007). En las categorías restantes, los estudiantes solo dieron como definición una descripción de su imagen evocada. Esto apoya lo dicho por Vinner (2002) respecto a que la actividad de los estudiantes está, en una mayoría de casos, basada solo en sus imágenes y no en las definiciones formales de los conceptos. Aunado a ello Acuña (2012) comenta que la definición de un objeto matemático es sustituida por la descripción de un ejemplo estereotipado que el estudiante asocia con la definición. Por último, ningún estudiante proporcionó alguna definición de ángulo que se correspondiera con las definiciones de ángulo dirigido u orientado.

Ítem 2. Conocer la imagen evocada del estudiante al hacer la representación gráfica del ángulo. Las representaciones gráficas de los estudiantes muestran un ángulo agudo, con su vértice ubicado del lado izquierdo, y del cual emanan dos rayos, uno de ellos horizontal. Ángulos con estas mismas características los 
podemos observar en la mayoría de las ilustraciones de los libros de texto que presentamos al inicio de este trabajo. Este hecho da lugar a considerar lo expresado por Vinner y Hershkowitz (1980) quienes comentan que los profesores y los libros de texto tienen una tendencia a dibujar ángulos con un rayo horizontal y como resultado de este hecho la imagen del concepto puede contener ángulos con un rayo horizontal, aunque puede suceder que dibujar este tipo de ángulos no signifique que son los únicos que forman parte de sus imágenes del concepto. Rösken y Rolka (2007) señalan que las figuras utilizadas como ejemplos de ángulos en la clase o en los libros de texto pueden llegar a formar parte de las imágenes de los estudiantes, y si estas se presentan sólo en ciertas posiciones estereotipadas, pueden volverse esenciales o determinantes al momento de resolver un problema.

Ítem 4. Conocer qué imagen evoca el estudiante para decidir si una figura dada es un ángulo o no. En el análisis de los resultados en el caso de la figura 12, se observa que el estudiante 1 reconoce su imagen de ángulo solamente en una parte de la figura (la punta), ignorando la totalidad de la figura. Este resultado es similar al reportado por Keiser (2004), donde señala que algunos estudiantes consideran que en el dibujo de las letras R o P puede haber ángulos al mirar intersecciones entre líneas y curvas. Por otro lado, para la figura 13 , se observa que la imagen del estudiante 2 está asociada con dos líneas diferentes con un punto común que forman una "abertura". Esta imagen lo llevó a decidir que la figura no era un ángulo al no visualizar en la figura 13 ninguna abertura. Este resultado se asemeja a lo encontrado en el trabajo de Keiser (2004), donde se menciona que las dificultades para concebir ángulos de $0^{\circ}, 180^{\circ}$ y $360^{\circ}$, y están relacionadas con la definición de ángulo que se está considerando.

Ítem 10. Averiguar la imagen evocada al momento de relacionar cada ángulo con su medida. En el caso del estudiante 3, posiblemente la posición en la que se encuentra el ángulo B le hizo pensar que este se situaba en un "tercer cuadrante", por ello trazó una prolongación del rayo horizontal que funcionaría como el sentido positivo de un eje $x$, tomándolo como lado inicial y desde ahí hacer la medición. Debe considerarse la posibilidad de que trazar la prolongación del rayo horizontal, se deba porque esto conduce a los estudiantes a una situación familiar donde están acostumbrados a medir ángulos desde el lado positivo del eje $x$. En el caso del estudiante 4, un ángulo se forma con dos segmentos diferentes. Al parecer la posición del segmento que ve le sugiere considerarlo como la parte negativa del eje $x$, y el rayo trazado por él, como su complemento, la parte positiva del eje $x$, desde donde él mide el ángulo. El hecho de que 
asignaran medidas diferentes a ángulos iguales colocados en distintas posiciones es similar a lo reportado en Browning, Garza-Kling y Sundling (2007), donde señalan que algunos estudiantes no reconocen que dos ángulos tienen la misma medida si están orientados en direcciones diferentes no estándar.

Todos los nuevos ángulos que construyen los estudiantes están en posición normal. Posiblemente, su imagen de ángulo (en posición normal) está construida a partir de ilustraciones o ejemplos como el que se muestra en Leithold (1992), donde los ángulos parecen tener el lado inicial sobre el eje positivo $x$ de un plano cartesiano, y estar en un primer cuadrante. Con esto concluimos nuestra discusión de resultados.

\section{CONCLUSIONES Y REFLEXIONES FINALES}

En nuestra investigación nos hemos planteado las preguntas siguientes: ¿qué definiciones tienen los estudiantes del ángulo al iniciar la enseñanza superior? ¿Qué imágenes evocan del ángulo y su medida, al contestar ítems relacionados con estos conceptos? Encontramos una amplia variedad en las definiciones personales de ángulo de los estudiantes. Observamos que la imagen evocada por la mayoría de los estudiantes al hacer una representación gráfica del ángulo está fuertemente conectada a una figura estereotipada (un ángulo agudo, con su vértice ubicado del lado izquierdo del cual emanan dos rayos, con un rayo horizontal). Otro resultado está relacionado con ángulos colocados en distintas posiciones, donde algunos estudiantes utilizan parcialmente las figuras de ángulo dadas y evocando otros conceptos matemáticos, construyen nuevos ángulos para los cuales estiman o relacionan con una medida. Como los resultados lo indican algunas de las dificultades identificadas en estudiantes de niveles elementales persisten en niveles más avanzados.

Un resultado interesante es el caso de la figura 12, donde el estudiante 1 mira un ángulo entre líneas y curvas. Cuando el estudiante dice que la curva en algún momento tiene un comportamiento de línea recta al hacerle un zoom y ésta se conecta con la recta dada, él está indicando, de manera intuitiva, la forma en que se define el ángulo entre dos curvas en Cálculo (como el ángulo entre sus tangentes), y que será aprovechado así en dicho curso. Estas ideas intuitivas podrían explotarse con estudiantes que tienen alguna familiaridad con el uso de algún software de Geometría dinámica, para darle sentido a la definición posterior de ángulo entre dos curvas. 
En resumen, las imágenes evocadas por los estudiantes son congruentes con lo señalado en Vinner (2002), en cuanto a que la definición se vuelve inactiva, y los estudiantes responden basándose en su imagen del concepto. Además, de que los estudiantes evocan diferentes imágenes. Estas imágenes dependen del problema a resolver, es decir, no poseen una única imagen del concepto.

Según Driver, Guesne y Tiberghien (1985) una estrategia que permite adaptar mejor la enseñanza consiste en tener en cuenta las ideas previas de los estudiantes, puesto que en ocasiones algunos conceptos se consideran obvios y se dan por conocidos al planificar el curso. Además, las ideas de los estudiantes sugieren que incluso nociones aparentemente sencillas pueden no ser captadas de manera adecuada. La incomprensión de estas ideas fundamentales puede, en tal caso, llevar a posteriores y más serios problemas de aprendizaje. Por lo tanto, en nuestro tema, el profesor podría tomar en cuenta las definiciones e imágenes mostradas en este estudio para el diseño de actividades que permitan al estudiante tener un mejor desempeño y tener presente que los estudiantes podrían evocar nuevos conceptos y sus imágenes, para contestar la tarea propuesta. También consideramos importante compartir con los estudiantes el hecho de que existe una definición de ángulo para geometría y otra para trigonometría (las cuales serán elegidas, según convenga, entre la variedad de definiciones encontradas en los libros de texto), al ser la definición de ángulo dada en trigonometría el resultado de una evolución y generalización tal y como se observa en la historia misma del concepto. Esta evolución no se detiene en la trigonometría, pues tenemos además el concepto de ángulo entre curvas, que se utiliza en Cálculo y en la geometría hiperbólica.

Hay que tomar en cuenta que la muestra que se utilizó en este estudio fueron estudiantes con un mayor dominio de las matemáticas, sin embargo encontramos dificultades. Si este estudio se llevara a cabo con estudiantes de otras carreras universitarias, seguramente se encontrarían nuevos hallazgos. Recordemos que la importancia de estos hallazgos radica en ofrecer a los profesores información para la elección de los conceptos que enseñarán y para planear sus actividades de aprendizaje. 


\section{REFERENCIAS}

Acuña, C. (2012). La visualización como forma de ver en matemáticas; un acercamiento a la investigación. Gedisa.

Akkoc, H. (2008). Pre-service mathematics teachers' concept images of radian. International Journal of Mathematical Education in Science and Technology, 39(7), 857-878. doi: 10.1080/00207390802054458

Ayres, F. Moyer, R. (1991). Trigonometría (2da. ed.). México: McGraw-Hill Interamericana. Baldor, A. (1983). Geometría Plana y del Espacio y Trigonometría. Publicaciones Cultural.

Browning, C., Garza-Kling, G. y Sundling, E. (2007). What's your angle on angles? Teaching Children Mathematics, 14(5), 283-287.

Driver, R., Guesne, E. y Tiberghien, A. (1985). Las ideas de los niños y el aprendizaje de las ciencias. En R. Driver, E. Guesne y A. Tiberghien (Eds.), Las Ideas científicas en la infancia y en la Adolescencia. Morata/MEC.

Heath, T. (1956). The thirteen books of Euclid's Elements. Translated from the text of Heiberg with introduction and commentary (vol. I, 2nd. ed.). Dover.

Hilbert, D. (1971). The foundations of Geometry (L. Unger, Trans.) (2nd. ed.). La Salle, Open Court.

Keiser, J. M. (2004). Struggles with developing the concept of angle: Comparing sixth-grade students' discourse to the history of the angle concept. Mathematical Thinking and Learning, 6(3), 285-306. doi: 10.1207/s15327833mt10603_2

Leithold, L. (1992). El Cálculo con Geometría Analítica (6ta. ed). Harla.

Matos, J. (1990). The historical development of the concept of angle. The Mathematics Educator, 1(1), 4-11.

Matos, J. (1991). The historical development of the concept of angle (2). The Mathematics Educator, 2(1), 18-24.

Moise, E. y Downs, F. (1986). Geometría Moderna. Wilmington, Addison-Wesley Iberoamericana.

Pachuca, Y. (2014) Concepciones sobre el argumento de la función sen x en profesores del nivel medio superior y alumnos del nivel superior mexicano (Tesis de maestría). Cinvestav-IPN. México.

Rösken, B. y Rolka, K. (2007). Integrating intuition: The role of concept image and concept definition for students' learning of integral calculus. The Montana Mathematics Enthusiast, Monograph 3, 181-204.

Sampieri, R. H. (2018). Metodología de la investigación: las rutas cuantitativa, cualitativa y mixta. McGraw Hill. 
Spivak, M. (1993). Calculus: Cálculo infinitesimal. Reverté.

Tall, D. y Vinner, S. (1981). Concept image and concept definition in mathematics with particular reference to limits and continuity. Educational Studies in Mathematics, 12(2), 151-169. doi:10.1007/BF00305619

Vinner, S. y Hershkowitz, R. (1980). Concept images and common cognitive paths in the development of some simple geometrical concepts. In R. Karplus (Ed.), Proceedings of the Fourth International Conference for the Psychology of Mathematics Education (pp. 177-184). University of California, Lawrence Hall of Science.

Vinner, S. (1983). Concept definition, concept image and the notion of function. International Journal of Mathematical Education in Science and Technology, 14(3), 293-305. doi: 10.1080/0020739830140305

Vinner, S. (2002). The role of definitions in the teaching and learning of mathematics. In D. Tall (Ed.), Advanced Mathematical Thinking (pp. 65-81). Kluwer. doi: 10.1007/0-306-47203-1_5

Vinner, S. (2011). The role of examples in the learning of mathematics and in everyday thought processes. ZDM The International Journal of Mathematics Education, 43(2), 247-256. doi:10.1007/s11858-010-0304-3

White, P. y Mitchelmore, M. (2003). Teaching angles by abstraction from physical activities with concrete materials. In N. A. Pateman, B. J. Dougherty y J. Zilliox (Eds.), Proceedings of the 27th Annual Conference of the International Group for the Psychology of Mathematics Education, (vol. 4, pp. 403-410). PME.

YANIRA PACHUCA HERRERA

Dirección: Departamento de Matemática Educativa-Cinvestav IPN

Av. Instituto Politécnico Nacional 2508, Col. San Pedro Zacatenco

C.P. 07360, México, Ciudad de México.

Teléfonos: (52)+(55)-57-47-38-15 


\section{ANEXO}

\section{CUESTIONARIO}

\section{TEMA: EL ÁNGULO Y SU MEDIDA}

Instrucciones: Lee cuidadosamente cada uno de los siguientes ítems y contesta de la forma más clara posible. Tus respuestas deben ser con pluma. Si de alguno de los ítems no conoces su respuesta indica brevemente por qué.

1. Escribe la definición de ángulo.

2. Dibuja un ángulo y señala cada una de sus partes.

4. Determina la o las figuras que consideres ángulos. (TÁCHALOS)
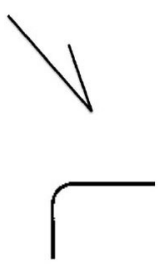
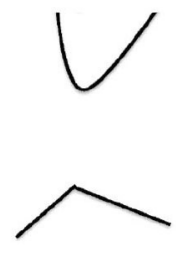
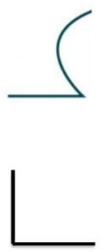
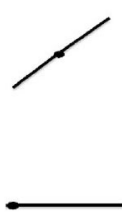

10. Relaciona cada ángulo con su medida. (Puedes relacionar las figuras con más de una opción)
a) Ángulo menor a
b) Ángulo mayor de
c) Ángulo mayor a
d) Ángulo igual a
e) Ángulo igual a
f) Ángulo igual a
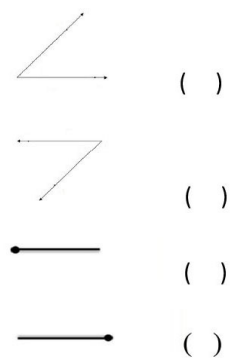